\title{
Theory of Optical-Filtering Enhanced Slow and Fast Light Effects in Semiconductor Optical Waveguides
}

Chen, Yaohui; Xue, Weiqi; Öhman, Filip; Mørk, Jesper

Published in:

Journal of Lightwave Technology

Link to article, DOI:

10.1109/JLT.2008.2004720

Publication date:

2008

Document Version

Publisher's PDF, also known as Version of record

Link back to DTU Orbit

Citation (APA):

Chen, Y., Xue, W., Öhman, F., \& Mørk, J. (2008). Theory of Optical-Filtering Enhanced Slow and Fast Light Effects in Semiconductor Optical Waveguides. Journal of Lightwave Technology, 26(23), 3734-3743.

https://doi.org/10.1109/JLT.2008.2004720

\section{General rights}

Copyright and moral rights for the publications made accessible in the public portal are retained by the authors and/or other copyright owners and it is a condition of accessing publications that users recognise and abide by the legal requirements associated with these rights.

- Users may download and print one copy of any publication from the public portal for the purpose of private study or research.

- You may not further distribute the material or use it for any profit-making activity or commercial gain

- You may freely distribute the URL identifying the publication in the public portal 


\title{
Theory of Optical-Filtering Enhanced Slow and Fast Light Effects in Semiconductor Optical Waveguides
}

\author{
Yaohui Chen, Weiqi Xue, Filip Öhman, and Jesper Mørk
}

\begin{abstract}
A theoretical analysis of slow and fast light effects in semiconductor optical amplifiers based on coherent population oscillations and including the influence of optical filtering is presented. Optical filtering is shown to enable a significant increase of the controllable phase shift experienced by an intensity modulated signal traversing the waveguide. The theoretical model accounts for recent experimental results and is used to analyze and interpret the dependence on material and device parameters. Furthermore analytical approximations are derived using a perturbation approach and are used to gain a better physical understanding of the underlying phenomena.
\end{abstract}

Index Terms-Coherent population oscillations (CPOs), fast light, four-wave mixing (FWM), microwave photonics, optical filtering, semiconductor optical amplifiers (SOAs), slow light.

\section{INTRODUCTION}

$\mathbf{I}$ $\mathrm{N}$ THE PAST few years, the possibility of controlling the group velocity of an optical signal propagating in a solid-state device has attracted increasing attention due to the possibilities of realizing compact devices for signal processing, such as all-optical buffering and phase shifters for microwave photonics. Semiconductor-based devices, with their well-developed fabrication technology and the possibilities for integration with other functionalities, are important candidates for practical applications. In the now classical demonstrations of light slow-down by Hau et al. [1], the phenomenon of electromagnetically induced transparency (EIT) in cold atoms was used to achieve the strong dispersion of the refractive index effect which lies at the heart of light-speed control. Semiconductor quantum dots (QDs) seem to be a natural choice to pursue light slow-down in semiconductor media with their discrete electronic levels [2]. However, since EIT relies on a quantum mechanical coherence among levels and dephasing times in semiconductors are short and, at the same time, present-day technology leads to QDs with large size dispersion, there are significant challenges to overcome for realizing practical light-speed control in semiconductors based on EIT [2], [3].

Instead, the effect of coherent population oscillation (CPO) [4] may provide a realistic alternative. This effect relies on the beating between two externally injected laser beams, or a single

Manuscript received May 02, 2008; revised August 06, 2008. Current version published January 28, 2009. This work was supported by the Danish Research Councils through the project QUEST.

The authors are with DTU Fotonik, Department of Photonics Engineering, Technical University of Denmark, DK-2800 Kgs. Lyngby, Denmark (e-mail: ych@com.dtu.dk; xue@com.dtu.dk; filip.ohman@syntune.com; jm@com.dtu. $\mathrm{dk})$.

Color versions of one or more of the figures in this paper are available online at http://ieeexplore.ieee.org.

Digital Object Identifier 10.1109/JLT.2008.2004720 intensity-modulated laser beam with sidebands, which leads to oscillations of the electron density in the medium, which in turn alter the effective index seen by the optical signal. In general, the effect can therefore be described as a wave mixing phenomenon, where interactions between the frequency components are mediated by the complex susceptibility, which in semiconductor structures has contributions from various carrier dynamical processes [5], [6].

It has by now been experimentally demonstrated that light-speed control can be realized by CPO effects in a number of different semiconductor structures, i.e., bulk, quantum well (QW) or QD semiconductor optical amplifiers (SOAs) [6]-[10], electro absorbers (EAs) [5], and integrated SOA-EA pairs [11], [12].

The slow and fast light effects are usually measured by considering the phase shift imposed upon the intensity-modulated envelope of an optical signal propagating through the device. When the modulation frequency is in the microwave region, such signals form the basis of microwave photonics [13], where a phase shifter is a much desired functionality. From an application point of view, it is thus of significant interest to realize an optically fed microwave phase shifter based on semiconductor devices with a variable phase shift range of $2 \pi$ at gigahertz frequencies. However, the maximum phase shift and bandwidth will be limited by different effects [14]. In both SOAs and EAs, the carrier lifetime sets an important limit [5], [6]. For absorbing media, the residual loss further limits the achievable delay [6]. The amplified spontaneous emission (ASE) limits the available SOA gain, and hence the phase change, in long amplifiers [15]. The concatenation of alternating gain and absorber sections is one previously presented way to alleviate some of these limitations [11], [12]. Here, we present a theoretical analysis of another promising method for increasing the achievable phase change and bandwidth.

In the practically important case where the input optical signal is a double-sideband signal generated by sinusoidal modulation of a laser beam, it can be shown that the refractive index dynamics plays no role in the observed phase shift [5]. The change in group velocity is in this case only controlled by the gain or absorption dynamics. Instead, using a single sideband excitation, in the form of a single sideband modulation or a modulated probe beam slightly detuned form a strong pump beam, as the input optical signal has been proposed as a way of increasing the phase shift by benefiting from refractive index dynamics [16], [17]. However, FWM leads to the generation of a conjugate sideband, which to a large extent cancels the refractive index dynamics effects after photodetection.

Recently, we proposed enhancing the phase shift and bandwidth by employing optical filtering before photodetection [19], 
and a maximum phase shift of $\sim 150$ degrees at $19-\mathrm{GHz}$ modulation frequency is achieved in a bulk SOA [20], corresponding to a several-fold increase of the absolute phase shift as well as achievable bandwidth. In [18], electrical filtering was employed after photodetection in order to suppress higher harmonics. In contrast, we are here filtering in the optical domain directly, thereby selectively suppressing beating components, and this is shown to lead to the observation of both phase delay (slow light) and phase advance (fast light) in the same device.

In this paper, we present a theoretical analysis of the optical filter-enhanced microwave phase shifting based on a wave mixing description of carrier dynamics in SOAs. The model well accounts for the experimental results presented [20]. A semi-analytical model is derived to better understand the roles played by the different physical effects contributing to the microwave phase shift induced upon propagation. The paper is structured as follows. In Section II, the general model and a semi-analytical approximation are presented. These models are validated by comparison to experiments and subsequently used for examining the dependence on important material and device parameters in Section III. A simple perturbation analysis is presented in Section IV in order to better understand the physical processes and finally the conclusions are drawn in Section V. Detailed derivations are presented in the Appendix.

\section{MODEL}

\section{A. Microwave Phase Shifter}

Fig. 1(a) shows a general schematic of a photonic based microwave phase shifter. A tunable microwave phase shift can be achieved by controlling the optical signal processor. As shown in Fig. 1(b), the phase and amplitude of the electric fields of the optical signal are altered by an active semiconductor waveguide device, for example an SOA, via different wave mixing processes. By controlling the operation conditions of the photonic components, for example the input power to the SOA or its bias current, the microwave phase shift can be controlled. In addition to the SOA, optical filtering is included as part of optical signal processing. By employing an optical notch filter, one of the sidebands can be blocked and the other transmitted. When the bandwidth of the notch filter is much narrower than the modulation frequency, it can be approximated as an ideal filter suppressing all power in the sideband and the corresponding beating terms in the photodetector. We have investigated three cases: 1) passing both sidebands, corresponding to the standard case considered so far without optical filtering; 2) suppressing the blue and passing the red-shifted sideband, and 3) suppressing the red and passing the blue-shifted sideband.

\section{B. Microwave Modulated Optical Signal}

A weakly sinusoidal intensity-modulated (IM) signal with electric field of the general form

$$
\begin{aligned}
E(t, z)= & \left(E_{0}(z)+E_{-1}(z) e^{i \Omega t}+E_{+1}(z) e^{-i \Omega t}\right) e^{-i\left(\omega_{0} t-k_{0} z\right)} \\
= & \left(\left|E_{0}(z)\right| e^{i \theta_{0}}+\left|E_{-1}(z)\right| e^{i \Omega t+i \theta_{-1}}\right. \\
& \left.+\left|E_{+1}(z)\right| e^{-i \Omega t+i \theta_{+1}}\right) e^{-i\left(\omega_{0} t-k_{0} z\right)}
\end{aligned}
$$

is assumed. Here, $\omega_{0}$ is the frequency of the optical carrier, $k_{0}$ is the propagation constant of the carrier, and $\Omega$ is the modulation frequency. $E_{0}, E_{-1}, E_{+1}$ are the corresponding complex

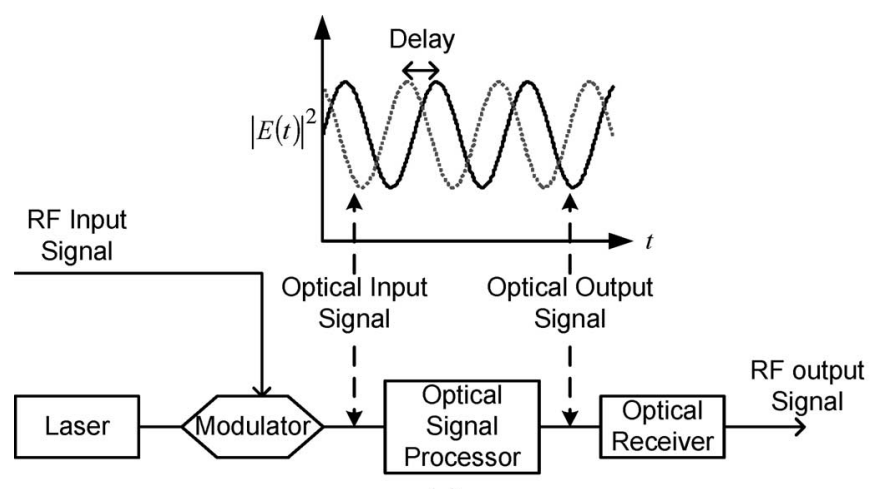

(a)

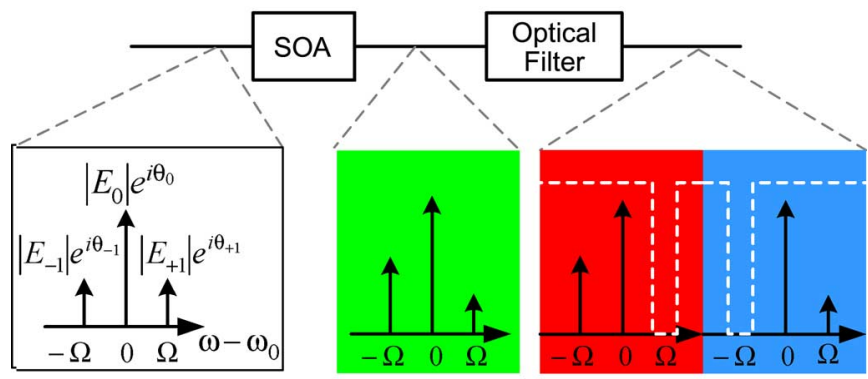

(b)

Fig. 1. (a) Schematic of a microwave phase shifter based on optical signal processing. (b) Optical signal processor based on SOA and pre-detection optical filtering. Optical output signal with i) no sideband removed, ii) with blue-shifted sideband removed, and iii) with red-shifted sideband removed.

amplitudes of the carrier, red-shifted sideband, and blue-shifted sideband. $\theta_{0}, \theta_{-1}$, and $\theta_{+1}$ are the corresponding optical spectral phases. For simplicity, the higher order sidebands are neglected.

When $\theta_{-1}-\theta_{0}=-\left(\theta_{+1}-\theta_{0}\right)$ and $\left|E_{-1}\right|=\left|E_{+1}\right|$, the optical signal is a pure amplitude modulated signal. When $\theta_{-1}$ $\theta_{0}=\pi-\left(\theta_{+1}-\theta_{0}\right)$ and $\left|E_{-1}\right| /\left|E_{0}\right|=\left|E_{+1}\right| /\left|E_{0}\right| \ll 1$, the optical signal can be approximated as a phase modulated signal. In practice, the output optical field after a general Mach-Zehnder electro-optical modulator contains amplitude modulation (AM) and phase modulation (PM) [21] and the spectral phase difference is different from the pure AM signal.

\section{Frequency Domain Modeling of SOA and Semi-Analytical Solution}

We theoretically analyze the slow and fast light effects in SOA structures due to CPO effects by using a wave mixing description [5], [6]. Wave mixing in active semiconductor waveguides has contributions from carrier density depletion, carrier heating $(\mathrm{CH})$, spectral hole burning (SHB), as well as two-photon absorption (TPA) and Kerr effects [22]. For the modulation frequency range investigated in this paper with a magnitude of the order of $\sim 1 / \tau_{s}$ (the inverse of carrier lifetime), i.e., up to some tens of $\mathrm{GHz}$, the dominating mechanism mediating the wave mixing is pulsation of carrier density [23], [24]. The ultrafast effects are therefore neglected, which is a reasonable approximation in the regime of moderate input optical power. Based on the weak modulation assumption, the wave mixing problem in this paper can be approximated and simplified as interactions between three optical waves 
[23], [24]. Gain saturation due to ASE is neglected, which is reasonable unless the input power is very low.

The propagation equations for the electric fields are

$$
\left\{\begin{array}{l}
\frac{\partial E_{0}}{\partial z}=\gamma_{0} E_{0} \\
\frac{\partial E_{-1}}{\partial z}=\gamma_{0} E_{-1}+\xi_{-1}\left(\left|E_{0}\right|^{2} E_{-1}+E_{0}^{2} E_{+1}^{*} e^{i \Delta k z}\right) \\
\frac{\partial E_{+1}}{\partial z}=\gamma_{0} E_{+1}+\xi_{+1}\left(\left|E_{0}\right|^{2} E_{+1}+E_{0}^{2} E_{-1}^{*} e^{i \Delta k z}\right)
\end{array}\right.
$$

with

$$
\begin{aligned}
\gamma_{0} & =\frac{1}{2}\left[g_{\mathrm{sat}}(1-i \alpha)-a_{\mathrm{int}}\right] \\
g_{\mathrm{sat}} & =\frac{\Gamma g_{0}}{1+S} \\
S & =\frac{\left|E_{0}\right|^{2}+\left|E_{-1}\right|^{2}+\left|E_{+1}\right|^{2}}{P_{\mathrm{sat}}} \\
\xi_{ \pm 1} & =-\frac{1}{2} \frac{g_{\mathrm{sat}}}{P_{\mathrm{sat}}} \frac{1-i \alpha}{1+S \mp i \Omega \tau_{s}} .
\end{aligned}
$$

Here, $\gamma_{0}$ corresponds to the first-order susceptibility with saturated modal gain $g_{\mathrm{sat}}$, linewidth enhancement factor $\alpha$, and internal waveguide loss $a_{\text {int }} . \Gamma g_{0}$ is the unsaturated modal gain, $S$ is the normalized $\mathrm{CW}$ optical power, and $P_{\text {sat }}$ is saturation power. $\xi_{ \pm 1}$ corresponds to the complex third-order susceptibility at the sidebands. $\tau_{s}$ is the carrier lifetime. $\Delta k$ is the phasemismatching factor induced by background material dispersion and waveguide dispersion. The phase-matching condition $\Delta k=$ 0 is fulfilled to a good approximation at the low detuning frequencies considered here [24].

Equation (2) is nonlinear and can be solved numerically to directly provide the output signal from the SOA. However, in order to highlight the physical effects of the microwave phase shifting, and especially the effect of the filter, we derive a more transparent semi-analytical solution, which is subsequently, in Section IV, considered in the perturbation treatment. The amplitudes $E_{-1}$ and $E_{+1}$ are assumed to be small and only firstorder terms are included, consistently with the assumption of small-signal modulation. Similarly, gain saturation is governed by the carrier (pump) signal, corresponding to $S \approx\left|E_{0}\right|^{2} / P_{\text {sat }}$. By assuming, without loss of generality, the input electric field $E_{0}(0)$ to be real and defining the input conditions as

$$
\left\{\begin{array}{l}
u(0)=E_{+1}(0)+E_{-1}^{*}(0) \\
v(0)=E_{+1}(0)-E_{-1}^{*}(0)
\end{array}\right.
$$

then a general analytical solution to (2) can be obtained for an SOA with given device length $L$ (details in Appendix)

$$
\left\{\begin{array}{l}
E_{0}(L)=E_{0}(0) e^{F(L)} \\
E_{+1}(L)=\widetilde{E}_{+1}(L) e^{F(L)} \\
\quad=e^{F(L)}\left\{\frac{1}{2} v(0)+\frac{1}{2} u(0)\left[e^{H(L)}+i \alpha\left(1-e^{H(L)}\right)\right]\right\} \\
E_{-1}^{*}(L)=\widetilde{E}_{-1}^{*}(L) e^{F(L)^{*}} \\
\quad=e^{F(L)^{*}}\left\{-\frac{1}{2} v(0)+\frac{1}{2} u(0)\left[e^{H(L)}-i \alpha\left(1-e^{H(L)}\right)\right]\right\}
\end{array}\right.
$$

The common complex amplification factor $F(L)$ is

$$
F(L)=\int_{0}^{L} \gamma_{0} d z=\int_{S(0)}^{S(L)} \frac{\gamma_{0}}{\left(\gamma_{0}+\gamma_{0}^{*}\right) S^{\prime}} d S^{\prime}
$$

and the gain grating related complex amplification factor $H(L)$ is

$$
\begin{aligned}
H(L) \equiv & \gamma_{1}+i \beta_{1} \\
= & P_{\text {sat }} \int_{0}^{L}\left(\xi_{+1}+\xi_{-1}^{*}\right) S d z \\
= & P_{\text {sat }} \int_{S(0)}^{S(L)} \frac{\left(\xi_{+1}+\xi_{-1}^{*}\right) S^{\prime}}{\left(\gamma_{0}+\gamma_{0}^{*}\right) S^{\prime}} d S^{\prime} \\
= & -\int_{S(0)}^{S(L)} \frac{g_{\text {sat }}}{g_{\text {sat }}-a_{\text {int }}} \frac{1+S^{\prime}}{\left(1+S^{\prime}\right)^{2}+\left(\Omega \tau_{s}\right)^{2}} d S^{\prime} \\
& -i \int_{S(L)} \frac{g_{\text {sat }}}{g_{\text {sat }}-a_{\text {int }}} \frac{\Omega \tau_{s}}{\left(1+S^{\prime}\right)^{2}+\left(\Omega \tau_{s}\right)^{2}} d S^{\prime} .
\end{aligned}
$$

Here, $\gamma_{1}$ and $\beta_{1}$ indicate the real and imaginary part of $H(L)$, respectively. The integrals are determined by the input and output optical power $S$ of the SOA, which can be calculated by solving the $\mathrm{CW}$ optical power propagation equation for the SOA numerically [5], [6]. Based on the wave mixing description and linear treatment shown in (5), $F(L)$ can be considered as a complex amplification to the $\mathrm{CW}$ signal due to the first-order susceptibility and $H(L)$ can be considered as the spatial integral of the spatially varying gain grating due to third-order susceptibility, which is implicitly determined by the spatial variation of the $\mathrm{CW}$ optical power $S$ as well as the carrier lifetime $\tau_{s}$ along the waveguide. The refractive index grating is quantified by a non-zero linewidth enhancement factor $\alpha$.

For a given SOA, the values of functions $F(L)$ and $H(L)$ can be modified by changing the unsaturated modal gain (electrical bias/current control), input CW optical power (optical intensity control) and modulation frequency (frequency detuning). In the following sections, numerical results refer to the model based on (2) and semi-analytical results refer to the model based on (5).

\section{Photodetection and Optical Filtering}

Due to the frequency beating between the optical waves in the photodetector, the photocurrent at modulation frequency $\Omega$ is proportional to the corresponding complex ac term $P_{\mathrm{AC}}$ of $E E^{*}$. For the microwave signal, a time delay $\Delta t$ will introduce a phase change to $\left|P_{\mathrm{AC}}\right| e^{-i \Omega(t-\Delta t)}$ which is positive for a time delay and negative for a time advance.

The absolute microwave phase is determined by $\arg \left\{P_{\mathrm{AC}}\right\}$, which can be tuned through different control schemes. The microwave phase shift $\Delta \varphi=\arg \left\{P_{\mathrm{AC}}\right\}-\left.\arg \left\{P_{\mathrm{AC}}\right\}\right|_{\text {reference }}$ is stated relative to a reference situation, e.g., corresponding to $|H(L)| \approx 0$. In the following, we separately write down the expressions for the ac power for the different cases of optical filtering.

\section{1) Without optical filtering before photodetection}

Without the notch filter the carrier and both sidebands are detected. The resulting ac power is

$$
\begin{aligned}
P_{\mathrm{AC}}(L) & =2\left(E_{0}^{*}(L) E_{+1}(L)+E_{0}(L) E_{-1}^{*}(L)\right) \\
& =\frac{2\left|E_{0}(L)\right|^{2}}{E_{0}(0)} u(0) e^{H(L)} .
\end{aligned}
$$


The microwave phase shift induced by the SOA is determined by

$$
\begin{aligned}
\Delta \varphi & =\arg \left\{u(0) e^{H(L)}\right\}-\left.\arg \left\{u(0) e^{H(L)}\right\}\right|_{|H(L)| \approx 0} \\
& \approx \arg \left\{u(0) e^{H(L)} \cdot u(0)^{*}\right\} .
\end{aligned}
$$

\section{2) Red-shifted sideband}

With the notch filter blocking the blue-shifted sideband before photodetection only the red shifted sideband and the carrier are detected. The resulting ac power is

$$
\begin{aligned}
P_{\mathrm{AC}}(L)=2\left(E_{0}(L) E_{-1}^{*}(L)\right) \\
=\frac{2\left|E_{0}(L)\right|^{2}}{E_{0}(0)}\left\{-\frac{1}{2} v(0)+\frac{1}{2} u(0)\right. \\
\left.\times\left[e^{H(L)}-i \alpha\left(1-e^{H(L)}\right)\right]\right\} .
\end{aligned}
$$

The microwave phase shift induced by the SOA is determined by

$$
\begin{aligned}
& \Delta \varphi_{1}=\arg \{\left.-\frac{1}{2} v(0)+\frac{1}{2} u(0)\left[e^{H(L)}-i \alpha\left(1-e^{H(L)}\right)\right]\right\} \\
&-\arg \left\{-\frac{1}{2} v(0)+\frac{1}{2} u(0)\right.\left.\times\left[e^{H(L)}-i \alpha\left(1-e^{H(L)}\right)\right]\right\}\left.\right|_{|H(L)| \approx 0} \\
& \approx \arg \left\{\left\{-\frac{1}{2} v(0)+\frac{1}{2} u(0)\left[e^{H(L)}-i \alpha\left(1-e^{H(L)}\right)\right]\right\}\right. \\
&\left.\cdot\left[-\frac{1}{2} v(0)+\frac{1}{2} u(0)\right]^{*}\right\} .
\end{aligned}
$$

\section{3) Blue-shifted sideband}

With the notch filterblocking the red-shifted sideband before photodetection only the blue-shifted sideband and the carrier are detected. The resulting ac power is

$$
\begin{aligned}
P_{\mathrm{AC}}(L)= & 2\left(E_{0}^{*}(L) E_{+1}(L)\right) \\
=\frac{2\left|E_{0}(L)\right|^{2}}{E_{0}(0)} & \left\{\frac{1}{2} v(0)+\frac{1}{2} u(0)\right. \\
& \left.\times\left[e^{H(L)}+i \alpha\left(1-e^{H(L)}\right)\right]\right\} .
\end{aligned}
$$

The microwave phase shift induced by SOA is determined by

$$
\begin{aligned}
\Delta \varphi_{+1}=\arg \{ & \left.\frac{1}{2} v(0)+\frac{1}{2} u(0)\left[e^{H(L)}+i \alpha\left(1-e^{H(L)}\right)\right]\right\} \\
-\arg \{ & \frac{1}{2} v(0)+\frac{1}{2} u(0) \\
& \left.\times\left[e^{H(L)}+i \alpha\left(1-e^{H(L)}\right)\right]\right\}\left.\right|_{|H(L)| \approx 0} \\
\approx \arg \{ & \left\{\frac{1}{2} v(0)+\frac{1}{2} u(0)\left[e^{H(L)}+i \alpha\left(1-e^{H(L)}\right)\right]\right\} \\
& \left.\cdot\left[\frac{1}{2} v(0)+\frac{1}{2} u(0)\right]^{*}\right\} .
\end{aligned}
$$

Thus, based on the semi-analytical solution and different optical filtering schemes, the corresponding phase shift and ac power depends differently on the complex amplification

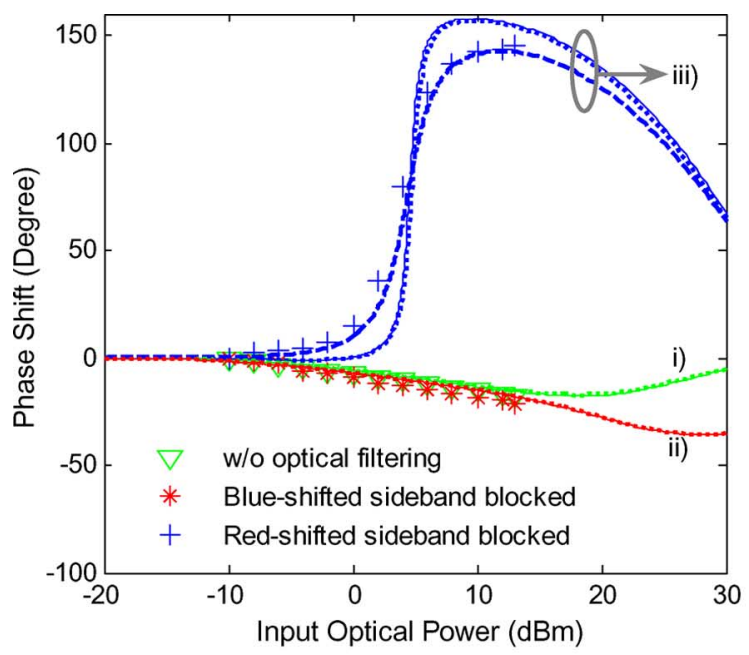

(a)

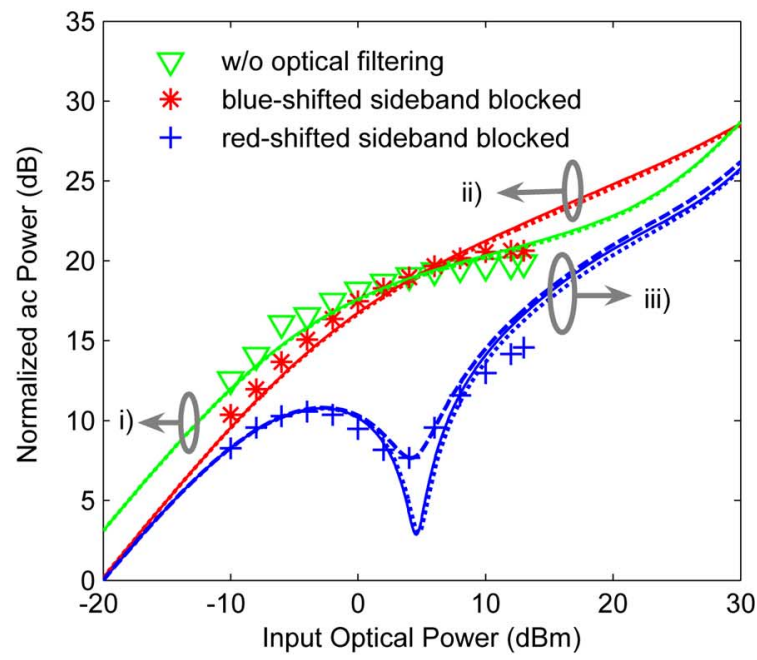

(b)

Fig. 2 (a) Phase shifts and (b) ac power change versus the input optical power in an SOA with different optical filtering schemes. The markers are experimental data taken on a bulk SOA at a modulation frequency of $19 \mathrm{GHz}$. The lines show the theoretical results for a pure AM input signal. The dotted lines are numerical results; the solid lines are semi-analytical results. The dashed line is a numerical result for an IM input signal with $\theta_{+1}-\theta_{0}=\theta_{-1}-\theta_{0}=0.1 \mathrm{rad}$. Main model parameters: $P_{\mathrm{sat}}=10 \mathrm{dBm}, \tau_{s}=100 \mathrm{ps}, \Gamma g_{0} L=5.75, \alpha=6$, $a_{\text {int }} L=2.75$.

$H(L)$, the linewidth enhancement factor $\alpha$ as well as the input optical signal $u(0), v(0)$. In particular we notice that for the usual case where both sidebands are detected the phase shift does not depend on the $\alpha$-factor,i.e., the dynamics of the refractive index does not influence the phase of the envelope. This is in agreement with [5] where it was shown also that this case can be analyzed by considering the dynamical effects of gain saturation. The effect of gain saturation also explains the time shifting of ultrashort pulses [25] in the limit where material dispersion can be neglected.

\section{RESUlTS}

\section{A. Comparison to Experimental Results}

In Fig. 2, we compare experimental results from [20] with the results of our theoretical model. The experiments were carried out using a bulk SOA and a fiber Bragg grating (FBG) notch 
filter with 0.1-nm bandwidth. Experimental (markers) and theoretical results (lines) are shown for the phase shift and the ac power change at a modulation frequency of $19 \mathrm{GHz}$ as a function of input optical power for a fixed SOA injection current. The phase is measured relative to the values at the minimum input optical power. The ac power in the calculations is normalized to agree with the experimental value at the minimum input optical power when blocking red-shifted sideband. As seen in the figure the theoretical results agree very well with the experimental data for both phase shift and ac power. Both the numerical solution (solid line) and the semi-analytical calculation (dotted lines) are presented. The small difference between numerical and semi-analytical results for a pure AM input signal indicates that the semi-analytical solution provides a very good approximation for the phase shift. In addition, Fig. 2 shows modeling results for an IM input signal with a small spectral phase difference between different frequency components (dashed lines), corresponding to a realistic non-ideal modulator with a small chirp similar to the one used in the experiment. The inclusion of the small chirp provides a better quantitative agreement with experimental data. As shown in Fig. 2(a), comparing with the conventional case without optical filtering, blocking the red-shifted or blue-shifted sideband leads to positive and negative phase changes, corresponding to slow light and fast light, respectively. Especially when the red-shifted sideband is blocked, about 150 degrees phase delay is achieved, which corresponds to a tenfold increase of the maximum phase shift obtained in the absence of optical filtering.

\section{B. Parameter Dependence}

As discussed previously, the microwave phase shift in the SOA plus filter is influenced by several parameters. Here, we numerically investigate the influence of the modulation frequency (carrier lifetime), linewidth enhancement factor, modal gain (internal loss), and waveguide length.

As shown in Fig. 3, the phase shift for a pure AM signal as a function of modulation frequency indicates that the modulation response regarding the phase shift can be enhanced by optical filtering. Here the situation of minimum input power at maximum modulation frequency, $|H(L)| \approx 0$, is chosen as the reference for phase shift and ac power. Especially when the red-shifted sideband is blocked, $\sim 100$ degree variable phase delay can be achieved for 20-dB optical power change over a large bandwidth $\sim 20 \mathrm{GHz}$. The sharp increase of the phase shift also corresponds to a dip in ac power. The result as a function of $\Omega \tau_{s}$ indicates that the modulation frequency can also be enhanced with a scale given by $1 / \tau_{s}$. With the assumption of a constant carrier lifetime in the device, it qualitatively explains the experimentally measured phase shift and relative ac power as a function of modulation frequency in [20].

The phase shift results for a pure AM signal at a modulation frequency of $10 \mathrm{GHz}$ as a function of the linewidth enhancement factor are shown in Fig. 4. When the $\alpha$-factor is zero, the employment of optical filtering has no influence on the phase shift as the refractive index dynamics is negligible. All of the cases hence give the same phase shift as seen in the Fig. 4. When the $\alpha$-factor increases, the constant phase advance value for the conventional case without optical filtering indicates the refractive index dynamics effects are cancelled after photodetection.

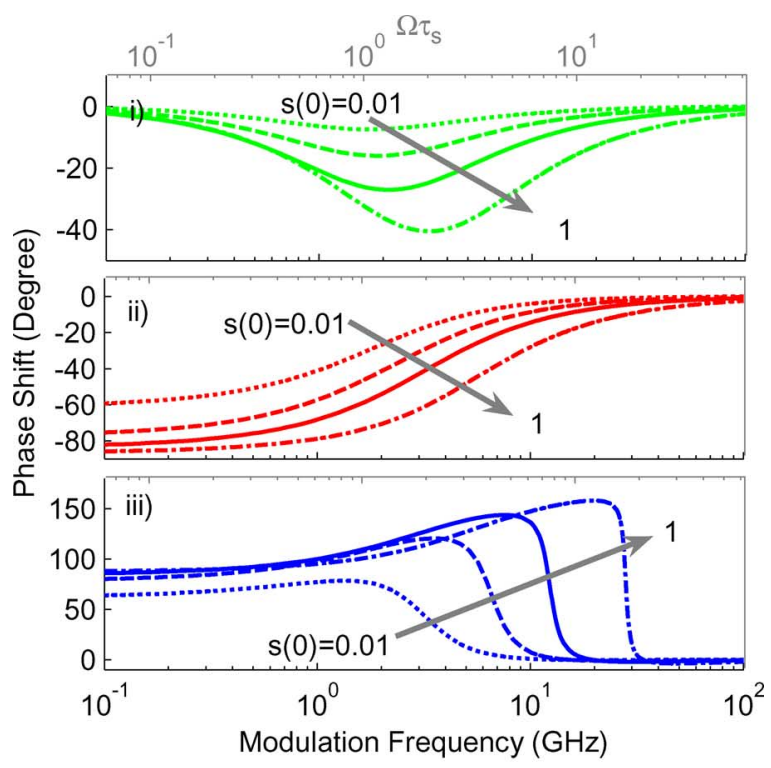

(a)

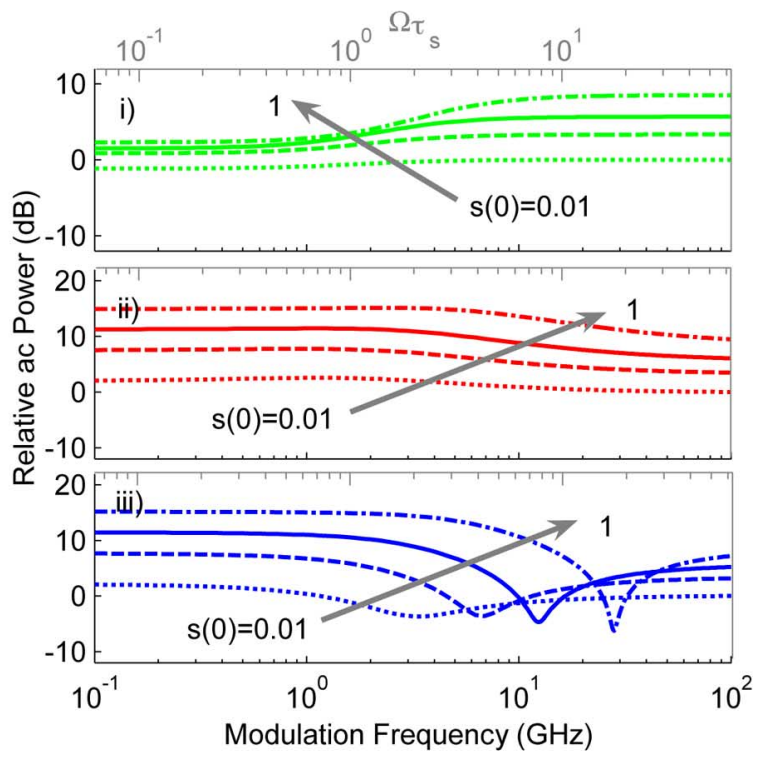

(b)

Fig. 3. (a) Phase shifts and (b) ac power change versus modulation frequency in an SOA with different optical filtering schemes. The numerical results are for a pure AM signal at different normalized input optical power $S(0): 0.01$ (dotted line), 0.03 (dashed line), 0.1 (solid line) and 1 (dash-dotted line). Main model parameters: $\tau_{s}=100 \mathrm{ps}, \Gamma g_{0} L=5.75, \alpha=6, a_{\mathrm{int}} L=2.75$. Phase shift and ac power are relative to the value with minimum input power at maximum modulation frequency $(|H(L)| \approx 0)$.

While the red-shifted sideband is blocked, the contribution from the refractive index dynamics turns the phase advance into a large delay. Moreover, comparing the results in Fig. 4(a) and (b), the gain grating and refractive index grating for the given modulation frequency are stronger in an SOA with the shorter carrier lifetime $\tau_{s}=50 \mathrm{ps}$ than the one with $\tau_{s}=100 \mathrm{ps}$. This also reveals that the magnitude of gain and refractive index grating depends on the product of modulation frequency and carrier lifetime shown in Fig. 3.

For bulk or QW semiconductor material with moderate $\alpha$-factor, the refractive index dynamics for a given input power is strong. For QD semiconductor material with a small $\alpha$-factor 


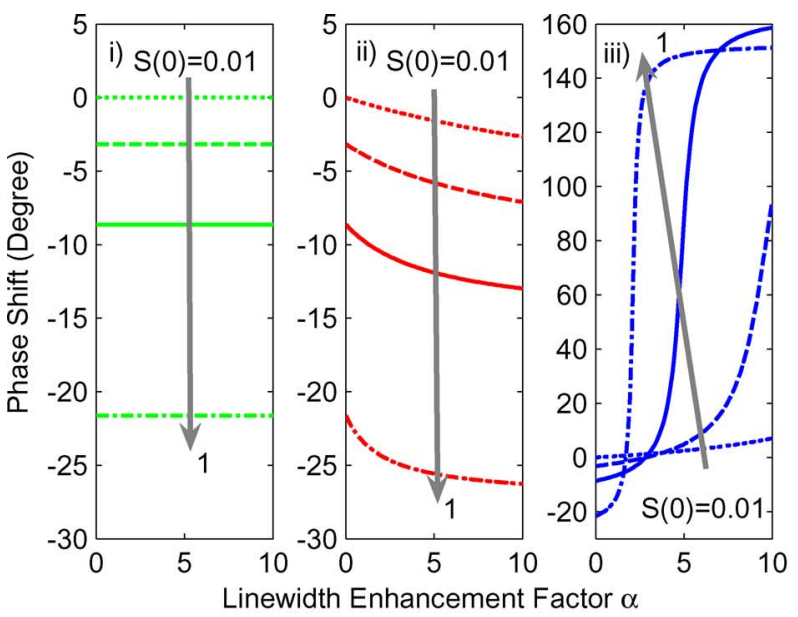

(a)

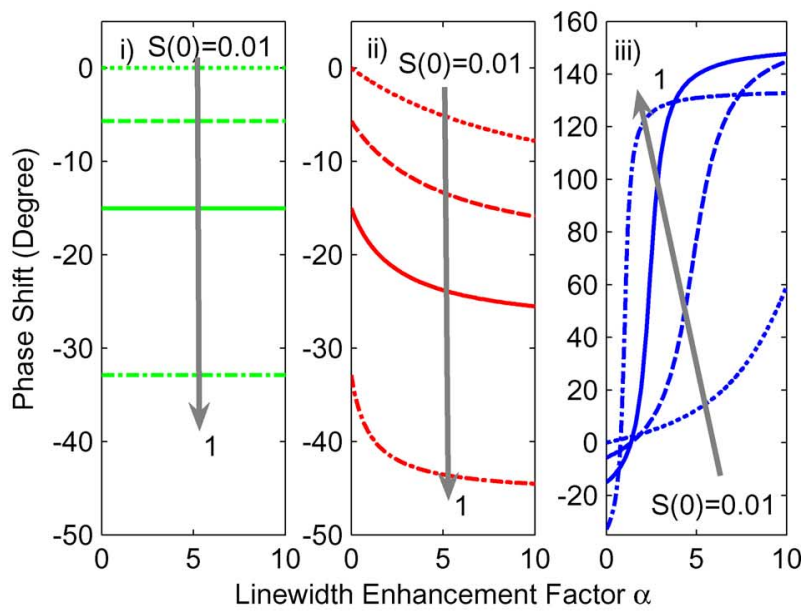

(b)

Fig. 4 Phase shifts versus linewidth enhancement factor a in an SOA with different optical filtering schemes for (a) $\tau_{s}=100 \mathrm{ps}$ and (b) $\tau_{s}=50 \mathrm{ps}$. The numerical results are for a pure AM input signal at a modulation frequency of $10 \mathrm{GHz}$ with different normalized input power $S(0): 0.01$ (dotted line), 0.03 (dashed line), 0.1 (solid line), and 1 (dash-dotted line). Main model parameters: $\Gamma g_{0} L=5.75, a_{\mathrm{int}} L=2.75$. Phase shift is relative to the value at minimum input optical power with zero value of $\alpha$.

the refractive index varies less but this might be compensated by a short carrier lifetime.

The phase shift results for a pure AM signal at a modulation frequency $10 \mathrm{GHz}$ as a function of modal gain for a 500- $\mu \mathrm{m}$-long active semiconductor waveguide are shown in Fig. 5. Here, we estimated the phase shifting in the absorption/gain regime with the assumption of constant carrier lifetime and saturation power, Although the absolute scaling of the phase shift will be different for realistic varying values of carrier lifetime and saturation power for different electrical bias/current control, the results qualitatively indicate the properties of phase delay/advance by electrical bias/current control. By tuning the SOA from the gain regime into absorption regime, the sign of the phase shift changes from negative (fast light) to positive (slow light) without optical filtering [9]. By blocking the blue-shifted sideband, we observe a large phase delay in the absorption regime and a phase advance in the gain regime. By blocking the red-shifted sideband, we observe a large phase delay in the gain regime and phase advance in

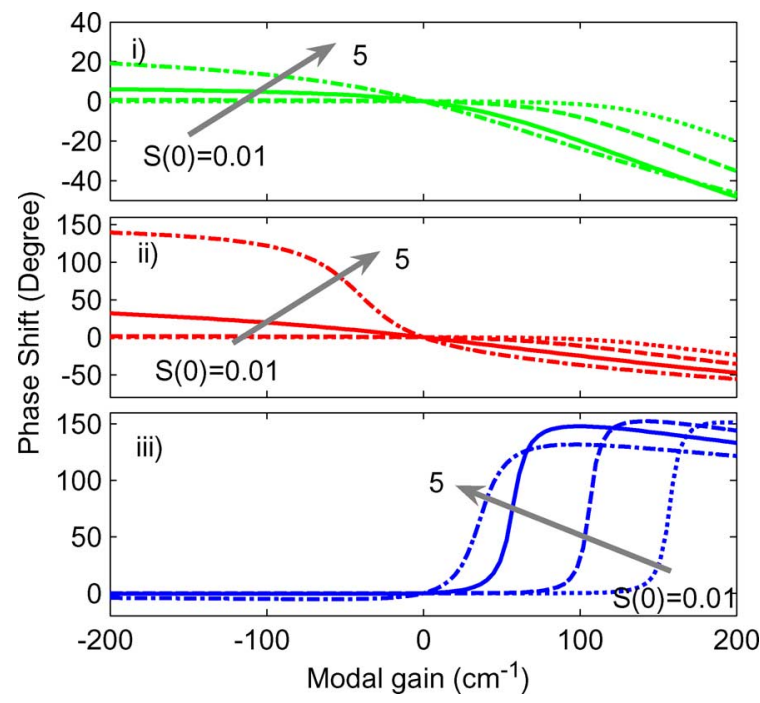

Fig. 5. Phase shift versus modal gain in an SOA with different optical filtering schemes. The numerical results for a pure AM input signal at a modulation frequency $10 \mathrm{GHz}$ with different normalized optical power $S(0)$ : 0.01 (dotted line), 0.1 (dashed line), 1 (solid line), and 5 (dash-dotted line). Main model parameters: $\tau_{s}=100 \mathrm{ps}, \alpha=6, \mathrm{~L}=500 \mu \mathrm{m}$. $\alpha_{\text {int }}=55 \mathrm{~cm}^{-1}$. Phase shift is relative to the value at zero modal gain.

the absorption regime. This flipping of the sign of the corresponding phase shift can be interpreted by the perturbation treatment in Section IV. The internal loss also influences the final phase shift as discussed in [6].

The phase shifts for a pure AM signal at a modulation frequency of $10 \mathrm{GHz}$ as a function of waveguide length of an SOA for different input optical power levels are presented in Fig. 6. For the conventional case without optical filtering, a long SOA device is expected to show larger device gain (unless limited by ASE) and thus enhance the variable phase shift range [6]. As shown in Fig. 6, for example, for the given input optical power range, 32 degree phase advance can be achieved through a 1000- $\mu \mathrm{m}$-long device comparing with 22 degree phase advance through a 500- $\mu \mathrm{m}$-long SOA. However, by blocking the redshifted sideband a much larger effect is obtained, about $\sim 150$ degree phase delay can be achieved in an SOA with the length of 200-600 $\mu \mathrm{m}$, corresponding to the moderate small-signal device gain of 5-15 dB. It indicates that the corresponding spatial integral of the refractive index grating (quantified by the large value of the $\alpha$-factor) over this length is significant enough to achieve a large variable phase change range. However, we should emphasize that our present model does not include ASE noise, which is expected to limit the gain and phase shift for long devices [15].

\section{PERTURBATION ANALYSIS AND DisCUSSION}

In order to gain a better understanding of the physical effects resulting in the large impact of optical filtering on the microwave phase shift, for example, the large phase change shown in Fig. 2 by tuning input optical power, it is helpful to further simplify the model. Therefore, we investigate the filter-assisted phase shift by perturbation analysis. For simplicity, we assume that the input optical signal satisfies $|u(0)|=u(0)=\varepsilon$ and $|v(0)|=0$ as an pure AM signal with amplitude $\varepsilon$ normalized to the carrier. Internal loss is also neglected. 


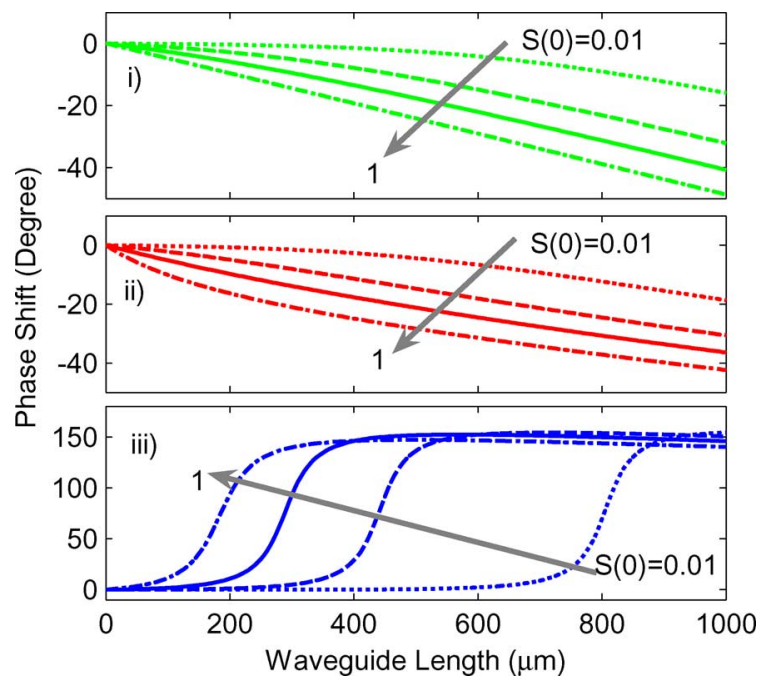

Fig. 6. Phase shift versus waveguide length in an SOA with different optical filtering schemes. The numerical results for a pure AM input signal at a modulation frequency $10 \mathrm{GHz}$ with different normalized optical power $\mathrm{S}(0)$ : 0.01 (dotted line), 0.1 (dashed line), 0.3 (solid line), and 1 (dash-dotted line). Main model parameters: $\left(\tau_{\mathrm{s}}=100 \mathrm{ps}, \alpha=6, . \Gamma \mathrm{g}_{0}=115 \mathrm{~cm}^{-1}\right.$, and $\alpha_{\mathrm{int}}=55 \mathrm{~cm}^{-1}$.) Phase shift is relative to the value at zero waveguide length.

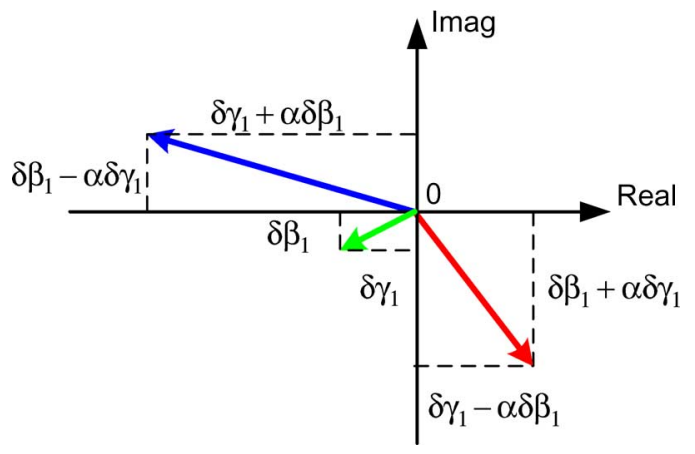

Fig. 7. Illustration of perturbation without spatial variation in an SOA.

\section{A. Perturbation Without Spatial Variation}

First, the enhancement of the phase shift by the optical filtering can be qualitatively understood from a perturbation treatment of the FWM in the limit of relatively small propagation length $\delta L$ [20]. This is equivalent to investigating the role of the gain grating and the refractive index grating as material response without spatial variation along the propagation coordinate.

We may obtain the electric fields after the amplification and interaction in the SOA based on (5) as

$$
\left\{\begin{array}{l}
\widetilde{E}_{+1}(\delta L)=\varepsilon\left(1+\delta \gamma_{1}+\alpha \delta \beta_{1}+i \delta \beta_{1}-i \alpha \delta \gamma_{1}\right) \\
\widetilde{E}_{-1}^{*}(\delta L)=\varepsilon\left(1+\delta \gamma_{1}-\alpha \delta \beta_{1}+i \delta \beta_{1}+i \alpha \delta \gamma_{1}\right)
\end{array}\right.
$$

where

$$
\left\{\begin{array}{l}
\delta \gamma_{1}=-g_{\mathrm{sat}} \frac{(1+S) S}{(1+S)^{2}+\left(\Omega \tau_{s}\right)^{2}} \delta L \\
\delta \beta_{1}=-g_{\mathrm{sat}} \frac{\Omega \tau_{s} S}{(1+S)^{2}+\left(\Omega \tau_{s}\right)^{2}} \delta L
\end{array} .\right.
$$

It is seen that both $\delta \gamma_{1}$ and $\delta \beta_{1}$ are negative for an SOA with positive gain. The perturbations with $\alpha$-factor, which indicate the sign of the corresponding phase changes, can be illustrated as a phasor diagram in Fig. 7.
When both sidebands are detected after the SOA, the phase shift becomes $\delta \varphi=\arg \left\{1+\delta \gamma_{1}+i \delta \beta_{1}\right\}$, indicating that the influence from the refractive index dynamics is cancelled.

When employing an optical filter to block the red-shifted sideband before detection, the phase shift, $\delta \varphi_{+1}=\arg \{1+$ $\left.\delta \gamma_{1}+\alpha \delta \beta_{1}+i\left(\delta \beta_{1}-\alpha \delta \gamma_{1}\right)\right\}$, includes a contribution $-\alpha \delta \gamma_{1}$, corresponding to an additional delay (positive phase shift) for positive $\alpha$-factor. If the blue-shifted sideband is blocked before detection, the phase shift $\delta \varphi_{-1}=\arg \left\{1+\delta \gamma_{1}-\alpha \delta \beta_{1}+i\left(\delta \beta_{1}+\right.\right.$ $\left.\left.\alpha \delta \gamma_{1}\right)\right\}$ instead contains a term $\alpha \delta \gamma_{1}$, corresponding to an additional advance.

When the semiconductor waveguide devices are biased in the absorption regime, the sign of the corresponding phase shift will be flipped as in Fig. 5.

\section{B. Perturbation Analysis Including Propagation Effects}

For an SOA device with given length $L$, the vision of perturbation analysis should be extended to take into account propagation effects. The optical pump power levels at the input and output of SOA satisfy the relation

$$
S(L)=S(0) T_{\text {sat }}
$$

Here, $T_{\text {sat }}=F(L)+F(L) *$ is the saturated value of the transmission. For a small perturbation of input power $\delta S$, the output power satisfies the relation

$$
\begin{aligned}
S(L)+\delta S_{\mathrm{out}} & =(S(0)+\delta S)\left(T_{\text {sat }}+\frac{\partial T_{\text {sat }}}{\partial S} \delta S\right) \\
& \approx S(0) T_{\mathrm{sat}}+\delta S\left(T_{\mathrm{sat}}+S(0) \frac{\partial T_{\mathrm{sat}}}{\partial S}\right)
\end{aligned}
$$

where $\delta S_{\text {out }}$ is the resulting perturbation of the output power. The corresponding change of $H(L)$ is

$$
\delta H(L) \equiv \delta \gamma_{1}(L)+i \delta \beta_{1}(L)=\left.H(L)\right|_{S(0)+\delta S}-\left.H(L)\right|_{S(0)}
$$

where

$$
\left\{\begin{array}{rl}
\frac{\delta \gamma_{1}(L)}{\delta S}= & P_{\text {sat }}\left[\frac{1+S(0)}{(1+S(0))^{2}+\left(\Omega \tau_{s}\right)^{2}}-\frac{1+S(L)}{(1+S(L))^{2}+\left(\Omega \tau_{s}\right)^{2}}\right. \\
& \left.\times\left(T_{\mathrm{sat}}+S(0) \frac{\partial T_{\mathrm{sat}}}{\partial S}\right)\right] \\
\frac{\delta \beta_{1}(L)}{\delta S}= & P_{\mathrm{sat}}\left[\frac{\Omega \tau_{s}}{(1+S(0))^{2}+\left(\Omega \tau_{s}\right)^{2}}-\frac{\Omega \tau_{s}}{(1+S(L))^{2}+\left(\Omega \tau_{s}\right)^{2}}\right. \\
& \left.\times\left(T_{\text {sat }}+S(0) \frac{\partial T_{\mathrm{sat}}}{\partial S}\right)\right]
\end{array} .\right.
$$

The electric fields after the amplification and interaction in the SOA are based on (5)

$$
\left\{\begin{array}{l}
\widetilde{E}_{+1}(L, \delta S)=\varepsilon\left[e^{H(L)}+i \alpha\left(1-e^{H(L)}\right)\right. \\
\left.\quad+e^{H(L)}\left(e^{\delta H(L)}-1\right)(1-i \alpha)\right] \\
\widetilde{E}_{-1}^{*}(L, \delta S)=\varepsilon\left[e^{H(L)}-i \alpha\left(1-e^{H(L)}\right)\right. \\
\left.+e^{H(L)}\left(e^{\delta H(L)}-1\right)(1+i \alpha)\right]
\end{array} .\right.
$$

The perturbations $e^{H(L)}\left(e^{\delta H(L)}-1\right)(1 \pm i \alpha)$ induced by the gain grating and the refractive index grating can alter the amplitude and phase of the corresponding electric fields destructively (or constructively) when the perturbations are out of phase (or in phase) compared with the complex amplification terms $e^{H(L)} \mp i \alpha\left(1-e^{H(L)}\right)$. As the destructive addition turns to the 
constructive one, the perturbations thus lead to a dip of the related ac power, as seen in Fig. 2.

Using (9), (11), and (13) for the different cases of filtering, the corresponding phase changes $\delta \varphi$ can be expressed as

$$
\delta \varphi=\arg \left\{|\Lambda|^{2}+\Delta\right\} .
$$

1) Without optical filtering before photodetection

$$
\left\{\begin{array}{l}
|\Lambda|^{2}=\left|e^{H(L)}\right|^{2} \\
\Delta=\left(e^{\delta H(L)}-1\right) \cdot\left|e^{H(L)}\right|^{2}
\end{array}\right.
$$

2) Red-shifted sideband

$$
\left\{\begin{array}{c}
|\Lambda|^{2}=\left|e^{H(L)}-i \alpha\left(1-e^{H(L)}\right)\right|^{2} \\
\Delta=\left(e^{\delta H(L)}-1\right)(1+i \alpha) \cdot e^{H(L)} \\
\cdot\left[e^{H(L)}-i \alpha\left(1-e^{H(L)}\right)\right]^{*}
\end{array}\right.
$$

3) Blue-shifted sideband

$$
\left\{\begin{array}{c}
|\Lambda|^{2}=\left|e^{H(L)}+i \alpha\left(1-e^{H(L)}\right)\right|^{2} \\
\Delta=\left(e^{\delta H(L)}-1\right)(1-i \alpha) \cdot e^{H(L)} \\
\cdot\left[e^{H(L)}+i \alpha\left(1-e^{H(L)}\right)\right]^{*}
\end{array}\right.
$$

Here, $|\Lambda|$ indicates the absolute value of the complex amplification without perturbation and $\Delta$ is the perturbation term. For a pure AM input signal, $|\Lambda|$ is correlated to the modulation index and ac power of the output optical signal into the photodetector.

To the first order in $\delta S$, we have

$$
\begin{array}{r}
\left(e^{\delta H(L)}-1\right)(1 \pm i \alpha) \approx\left(\delta \gamma_{1}(L) \mp \alpha \delta \beta_{1}(L)\right. \\
\left.+i \delta \beta_{1}(L) \pm i \alpha \delta \gamma_{1}(L)\right) .
\end{array}
$$

Here, we illustrate the perturbation results including propagation effects based on (21) as a phasor diagram in Fig. 8(a). The value of $\arg \{\Delta\}$ determines the sign of $\delta \varphi$ : A value between 0 and $\pi$ for $\arg \{\Delta\}$ introduces a positive phase change of $\delta \varphi$ (phase delay); a value between $-\pi$ and 0 for $\arg \{\Delta\}$ introduces a negative phase change of $\delta \varphi$ (phase advance). For a perturbation with unit amplitude $|\Delta|$, a larger phase change of $\delta \varphi$ is expected with a smaller $|\Lambda|$ value. By employing optical filtering, both $\Delta$ and $|\Lambda|$ are significantly influenced by $\alpha$-factor as well as $\delta \gamma_{1}(L), \delta \beta_{1}(L)$ and $H(L)$. In Fig. 8(b), a numerical example of (21) in an SOA is used to illustrate the complex situation, whereas only the electric field of blue-shifted sideband evolves from destructively to constructively. When the red-shifted sideband is blocked, the large phase change can thus be achieved around the dip of $|\Lambda|$. This also reveals the correlation between the large phase change and the dip in ac power seen in the numerical and experimental results as shown in Fig. 2.

When the device length is approaching zero, corresponding to $|H(L)| \approx 0$ and $|\Lambda| \approx 1$, this perturbation analysis is consistent with the simple interpretation of phase delay and advance without spatial variation in Section IV-A.

The perturbation analysis used here may also be used to analyze the role of the phase difference between different frequency components of the input optical signal, which will become increasingly important in practice. Such a phase difference may appear due to chirp in the modulator or dispersion in the optical path before the SOA. As far as modeling in the regime of weak modulation is concerned, a change of the phase difference does

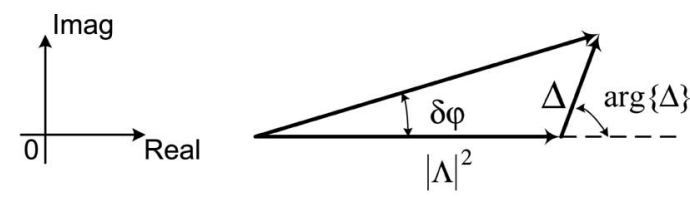

(a)

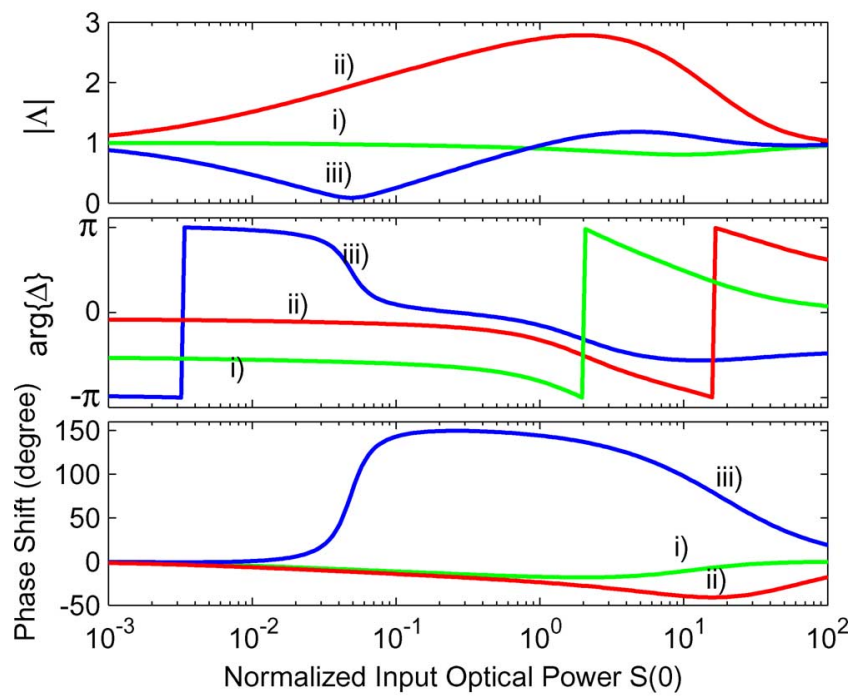

(b)

Fig. 8. (a) Illustration of the perturbation approach including propagation effects in an SOA. (b) Numerical example with different optical filtering schemes for a pure AM input signal as a function of normalized input optical power $S(0)$. $\left(\Gamma g_{0} L=5.75, \alpha_{\text {int }} L=0, \alpha=6\right.$, and $\left.\Omega \tau_{s}=12\right)$.

not change the gain grating or refractive index grating in the SOA from the case of pure AM signal, as seen in (7). However, the amplitude and phase of the electric fields are influenced by such a change of phase difference, based on (5), and thus the final filter-assisted phase delay or advance can be modified.

\section{CONCLUSION}

We have theoretically investigated the physical effects of CPO that contribute to microwave phase shifts in semiconductor optical waveguides, in particular analyzing the influence of optical filtering. The semi-analytical solution accounts for the evolution of the electric fields with complex amplification factors, which are spatial integrals of spatially varying gain and refractive index gratings. When optical filtering is included to selectively suppress a sideband of the optical signal before photodetection the refractive index grating becomes important and leads to the observation of both phase delay and phase advance. In the small signal regime, the strength of the gain grating is influenced by the input optical power (optical intensity control), modulation frequency/carrier lifetime (frequency detuning), modal gain/internal loss (electrical bias/current control). The strength of the refractive index grating is quantified by the $\alpha$-factor, which means that the influence of optical filtering can be increased significantly in QW and bulk devices, while QD devices with low $\alpha$-factor are expected to show a smaller influence from filtering. A large phase shift is achievable at higher modulation frequency with a moderate small signal device gain when properly exploiting optical filtering. Furthermore, the effects of phase difference between different frequency 
components of the input optical signal on the measured microwave phase shift adds the potential to further influence the filter-assisted phase shifts.

The phase-shifting effect induced by optical filtering is qualitatively explained by a simple perturbation approach. This approximate approach provides a simple physical explanation of the large difference, seen in both experiments and numerical simulations, between filtering the red and blue modulation sidebands.

\section{APPENDIX}

\section{GENERAL SOLUTION}

Based on (2), the propagation equation for $S$ is

$$
\frac{d S}{d z}=\left(\gamma_{0}+\gamma_{0}^{*}\right) S
$$

The propagation equation of $E_{0}$ can be transformed by substituting variable $z$ with normalized optical intensity $S$ as

$$
\frac{d E_{0}}{d S}-\frac{\gamma_{0}}{\left(\gamma_{0}+\gamma_{0}^{*}\right) S} E_{0}=0
$$

hich can be solved for $E_{0}$ to obtain

$$
\begin{aligned}
E_{0}(z)= & E_{0}(0) e^{F(z)} \\
F(z)= & \int_{S(0)}^{S(z)} \frac{\gamma_{0}}{\left(\gamma_{0}+\gamma_{0}^{*}\right) S^{\prime}} d S^{\prime} \\
= & \frac{1}{2} \frac{a_{\mathrm{int}}-\Gamma g_{0}+i \Gamma g_{0} \alpha}{a_{\mathrm{int}}-\Gamma g_{0}} \ln \left(\frac{S(z)}{S(0)}\right)-\frac{1}{2} \frac{i \Gamma g_{0} \alpha}{a_{\mathrm{int}}-\Gamma g_{0}} \\
& \times \ln \left(\frac{a_{\mathrm{int}}-\Gamma g_{0}+a_{\mathrm{int}} S(z)}{a_{\mathrm{int}}-\Gamma g_{0}+a_{\mathrm{int}} S(0)}\right) .
\end{aligned}
$$

Here, $S(z)$ and $S(0)$ are the output and input power of $S$, which can easily be related numerically by (A1). We can also keep the function $F(z)$ in the form of an integral over the propagation length $z$ in SOA with implicit relation between power $S$ and propagation length $z$ as:

$$
F(z)=\int_{0}^{z} \gamma_{0} d z^{\prime}
$$
$\tilde{E}_{ \pm 1}$

Here, we introduce the new normalized complex amplitude

$$
E_{ \pm 1}=\widetilde{E}_{ \pm 1} e^{F(z)}
$$

Inserting $\tilde{E}_{ \pm 1}$ into the propagation equations of $E_{ \pm 1}$, when $\left|e^{F(z)}\right| \neq 0$ and $\left|E_{0}(0)\right|=E_{0}(0)$, we have a new set of propagation equations of $\tilde{E}_{ \pm 1}$

$$
\left\{\begin{array}{l}
\frac{d \widetilde{E}_{-1}}{d z}=\xi_{-1}\left(\widetilde{E}_{-1}+\widetilde{E}_{+1}^{*}\right)\left|E_{0}(z)\right|^{2} \\
\frac{d \widetilde{E}_{+1}}{d z}=\xi_{+1}\left(\widetilde{E}_{+1}+\widetilde{E}_{-1}^{*}\right)\left|E_{0}(z)\right|^{2}
\end{array} .\right.
$$

By defining an extra pair of variables

$$
\left\{\begin{array}{l}
u=\widetilde{E}_{+1}+\widetilde{E}_{-1}^{*} \\
v=\widetilde{E}_{+1}-\widetilde{E}_{-1}^{*}
\end{array} .\right.
$$

We have

$$
\left\{\begin{array}{l}
\frac{d u}{d z}=\left(\xi_{+1}+\xi_{-1}^{*}\right)\left|E_{0}(z)\right|^{2} u \\
\frac{d v}{d z}=-i \alpha\left(\xi_{+1}+\xi_{-1}^{*}\right)\left|E_{0}(z)\right|^{2} u
\end{array} .\right.
$$

A propagation equation of the form

$$
\frac{d u}{d S}-P_{s a t} \frac{\left(\xi_{+1}+\xi_{-1}^{*}\right) S}{\left(\gamma_{0}+\gamma_{0}^{*}\right) S} u=0
$$

can be solved for $u, v$ to obtain

$$
\left\{\begin{array}{l}
u(z)=u(0) e^{H(z)} \\
v(z)=v(0)+i \alpha u(0)\left(1-e^{H(z)}\right)
\end{array}\right.
$$

where

$$
\begin{aligned}
& H(z) \equiv \gamma_{1}+i \beta_{1}=P_{\mathrm{sat}} \int_{S(0)}^{S(z)} \frac{\left(\xi_{+1}+\xi_{-1}^{*}\right) S^{\prime}}{\left(\gamma_{0}+\gamma_{0}^{*}\right) S^{\prime}} d S^{\prime} \\
& \gamma_{1}=\Gamma g_{0}\left[\frac{a_{\mathrm{int}} \tau_{s}^{2} \Omega^{2}}{\tau_{s} \Omega\left(\left(\Gamma g_{0}\right)^{2}+a_{\mathrm{int}}^{2} \tau_{s}^{2} \Omega^{2}\right)}\right. \\
& \times \operatorname{Arctan}\left(\frac{\Omega \tau_{s}(S(z)-S(0))}{\left(\Omega \tau_{s}\right)^{2}+(1+S(z))(1+S(0))}\right) \\
& +\frac{\Gamma g_{0}}{\left(\Gamma g_{0}\right)^{2}+a_{\text {int }}^{2} \tau_{s}^{2} \Omega^{2}} \\
& \times \ln \left(\frac{a_{\mathrm{int}}-\Gamma g_{0}+a_{\mathrm{int}} S(z)}{a_{\mathrm{int}}-\Gamma g_{0}+a_{\mathrm{int}} S(0)}\right) \\
& -\frac{1}{2} \frac{\Gamma g_{0}}{\left(\Gamma g_{0}\right)^{2}+a_{\mathrm{int}}^{2} \tau_{s}^{2} \Omega^{2}} \\
& \left.\times \ln \left(\frac{1+2 S(z)+S(z)^{2}+\tau_{s}^{2} \Omega^{2}}{1+2 S(0)+S(0)^{2}+\tau_{s}^{2} \Omega^{2}}\right)\right] \\
& \beta_{1}=\Gamma g_{0} \Omega \tau_{s} \\
& \times\left[-\frac{\Gamma g_{0}}{\tau_{s} \Omega\left(\left(\Gamma g_{0}\right)^{2}+a_{\mathrm{int}}^{2} \tau_{s}^{2} \Omega^{2}\right)}\right. \\
& \times \operatorname{Arctan}\left(\frac{\Omega \tau_{s}(S(z)-S(0))}{\left(\Omega \tau_{s}\right)^{2}+(1+S(z))(1+S(0))}\right) \\
& +\frac{a_{\text {int }}}{\left(\Gamma g_{0}\right)^{2}+a_{\text {int }}^{2} \tau_{s}^{2} \Omega^{2}} \\
& \times \ln \left(\frac{a_{\mathrm{int}}-\Gamma g_{0}+a_{\mathrm{int}} S(z)}{a_{\mathrm{int}}-\Gamma g_{0}+a_{\mathrm{int}} S(0)}\right) \\
& -\frac{1}{2} \frac{a_{\text {int }}}{\left(\Gamma g_{0}\right)^{2}+a_{\text {int }}^{2} \tau_{s}^{2} \Omega^{2}} \\
& \left.\times \ln \left(\frac{1+2 S(z)+S(z)^{2}+\tau_{s}^{2} \Omega^{2}}{1+2 S(0)+S(0)^{2}+\tau_{s}^{2} \Omega^{2}}\right)\right] \text {. }
\end{aligned}
$$

This result is equivalent to the semi-analytical solution for the time delay given in [6]. When the internal loss $a_{\text {int }}$ is neglected, (A13) is equivalent to the semi-analytical solution for the time delay given in [5].

The function $H(z)$ can be kept in the form of an integral over the propagation length $z$ with implicit relations between power $S$ and propagation length $z$

$$
H(z)=P_{\text {sat }} \int_{0}^{z}\left(\xi_{+1 u}+\xi_{-1}^{*}\right) S d z^{\prime} .
$$


Thus a general solution to the evolution of electric fields in SOA is

$$
\left\{\begin{array}{l}
E_{0}(z)=E_{0}(0) e^{F(z)} \\
E_{+1}(z)=\widetilde{E}_{+1}(z) e^{F(z)} \\
\quad=e^{F(z)}\left\{\frac{1}{2} v(0)+\frac{1}{2} u(0)\left[e^{H(z)}+i \alpha\left(1-e^{H(z)}\right)\right]\right\} \\
E_{-1}^{*}(z)=\widetilde{E}_{-1}^{*}(z) e^{F(z)^{*}} \\
\quad=e^{F(z)^{*}}\left\{-\frac{1}{2} v(0)+\frac{1}{2} u(0)\left[e^{H(z)}-i \alpha\left(1-e^{H(z)}\right)\right]\right\}
\end{array} .\right.
$$

\section{REFERENCES}

[1] C. Liu, Z. Dutton, C. H. Behroozi, and L. V. Hau, "Observation of coherent optical information storage in an atomic medium using halted light pulses," Nature, vol. 409, no. 6819, pp. 490-49, 2001.

[2] C. Chang-Hasnain, P.-C. Ku, J. Kim, and S.-L. Chuang, "Variable optical buffer using slow-light in semiconductor nanostructures," Proc. IEEE, vol. 91, p. 1884, 2003.

[3] P. K. Nielsen, H. Thyrrestrup, J. Mørk, and B. Tromborg, "Numerical investigation of electromagnetically induced transparency in a quantum dot structure," Opt. Exp., vol. 15, p. 6396, 2007.

[4] M. S. Bigelow, N. N. Lepeshkin, and R. W. Boyd, "Observation of ultra-slow light propagation in a ruby crystal at room temperature," Phys. Rev. Lett., vol. 90, no. 11, p. 113903, 2003.

[5] J. Mørk, R. Kjær, M. van der Poel, and K. Yvind, "Slow light in a semiconductor waveguide at gigahertz frequencies," Opt. Exp., vol. 13, pp. 8136-8145, 2005.

[6] H. Su, P. K. Kondratko, and S. L. Chuang, "Variable optical delay using population oscillation and four-wave-mixing in semiconductor optical amplifiers," Opt. Exp., vol. 14, pp. 4800-4807, 2006.

[7] P. Palinginis, S. Crankshaw, F. Sedgwick, E.-T. Kim, M. Moewe, and C. J. Chang-Hasnain, "Ultra-slow light $(<200 \mathrm{~m} / \mathrm{s})$ propagation in a semiconductor nanostructure," Appl. Phys. Lett., vol. 87, no. 17, p. 171102,2005

[8] H. Su and S. L. Chuang, "Room temperature slow and fast light in quantum dot semiconductor optical amplifiers," Applied Phys. Lett., vol. 88 , no. 6, p. 061102,2006

[9] P. K. Kondratko and S. L. Chuang, "Slow-to-fast light using absorption to gain switching in quantum-well semiconductor optical amplifier," Opt. Exp., vol. 15, pp. 9963-9969, 2007.

[10] C. Chang-Hasnain and S. L. Chuang, "Slow and fast light in semiconductor quantum-well and quantum-dot devices," J. Lightw. Technol., vol. 24, p. 4642, 2006.

[11] F. Öhman, K. Yvind, and J. Mørk, "Slow light in a semiconductor waveguide for true-time delay applications in microwave photonics," IEEE Photon. Technol. Lett., vol. 19, pp. 1145-1147, 2007.

[12] F. Öhman, K. Yvind, and J. Mørk, "Voltage-controlled slow light in an integrated semiconductor structure with net gain," Opt. Exp., vol. 14, pp. 9955-9962, 2006.

[13] J. Capmany, B. Ortega, D. Pastor, and S. Sales, "Discrete-time optical processing of microwave signals," J. Lightw. Technol., vol. 23, p. 702, 2005.

[14] R. S. Tucker, P.-C. Ku, and C. J. Chang-Hasnain, "Slow-light optical buffers: Capabilities and fundamental limitations," J. Lightw. Technol., vol. 23, p. 4046, 2005.

[15] T. Liu, K. Obermann, K. Petermann, F. Girardin, and G. Guekos, "Effect of saturation cased by amplified spontaneous emission on semiconductor optical amplifier performance," Opt. Lett., vol. 33, no. 24, pp. 2042-2043, 1997.

[16] A. Uskov and C. J. Chang-Hasnain, "Slow and superluminal light in semiconductor optical amplifiers," Electron. Lett., vol. 41, pp. 922-924, 2005.

[17] A. Uskov, F. Sedgwick, and C. J. Chang-Hasnain, "Delay limit of slow light in semiconductor optical amplifiers," IEEE Photon. Technol. Lett., vol. 18 , no. 6 , pp. $731-733,2006$.
[18] B. Pesala, Z. Cheng, A. V. Uskov, and C. J. Chang-Hasnain, "Experimental demonstration of slow and superluminal light in the semiconductor optical amplifiers," Opt. Exp., vol. 14, pp. 12968-12975, 2006.

[19] Y. Chen, F. Öhman, W. Xue, and J. Mørk, "Analysis of an effective optical filtering technique to enhance microwave phase shifts based on slow and fast light effects," in CLEO 2008 Conf. Dig., San Jose, CA.

[20] W. Xue, Y. Chen, F. Öhman, S. Sales, and J. Mørk, "Enhancing light slow-down in semiconductor optical amplifiers by optical filtering," Opt. Lett., vol. 21, pp. 1084-1086, 2008.

[21] Y. Shi, L. Yan, and A. E. Willner, "High-speed electrooptic modulator characterization using optical spectrum analysis," J. Lightw. Technol., vol. 21, no. 10, pp. 2358-2367, Oct, 2003.

[22] J. Mørk and A. Mecozzi, "Theory of ultrafast optical response of active semiconductor waveguides," J. Opt. Soc. Amer. B, vol. 13, pp. 1803-1816, 1996.

[23] G. P. Agrawal, "Population pulsations and nondegenerated four-wave mixing in semiconductor lasers and amplifiers," J. Opt. Soc. Amer. B, vol. 14, pp. 147-159, 1988.

[24] A. Uskov, J. Mørk, and J. Mark, "Wave mixing in semiconductor laser amplifiers due to carrier heating and spectral-hole burning," IEEE J. Quantum Electon., vol. 30, pp. 1769-1781, 1994.

[25] M. van der Poel, J. Mørk, and J. M. Hvam, "Controllable delay of ultrashort pulses in a quantum dot optical amplifier," Opt. Exp., vol. 13, pp. 8032-8037, 2005.

Yaohui Chen was born in Shanghai, China, in 1979. He received the B.Eng. and M.Eng. degree from Xi' an JiaoTong University, Xi' an, China, in 2001 and 2004, respectively, and the M.Sc. degree from the Technical University of Denmark, Lyngby, in 2007, all in electrical engineering. He is currently working toward the Ph.D. degree at DTU Fotonik, Technical University of Denmark.

His research interests are in carrier dynamics in nanostructured semiconductor materials, nanophotonics devices, and their applications.

Weiqi Xue received the B.Sc. degree in theoretical physics from Shanxi University, Taiyuan, China, in 2001 and the M. Eng. degree in physical electronics from Beijing University of Posts and Telecommunications, Beijing, China, in 2007. He is currently working toward the Ph.D. degree at the Technical University of Denmark, Lyngby, where he is involved in research on slow and fast light for applications in microwave photonics.

Filip Öhman received the M.Sc. degree in engineering from the Royal Institute of Technology (KTH), Sweden, in 2001 and the Ph.D. degree from the Technical University of Denmark Lynbgy, in 2005.

Between 2005 and 2008, he was a Postdoctoral Fellow with DTU Fotonik, Department of Photonics Engineering, Technical University of Denmark, where he was working with research on optical signal processing and noise in nonlinear semiconductor devices, in particular, investigating signal regeneration and applications of slow and fast light in microwave photonics using semiconductor optical amplifiers and electro-absorbers. He is currently with Syntune AB, Kista, Sweden.

Jesper Mørk received the M.Sc., Ph.D., and Dr. Techn. degrees from the Technical University of Denmark (DTU), Lyngby, in 1986, 1988, and 2003, respectively.

Since 2002, he has been a Professor in semiconductor devices for optical communication systems and, since 2008, he has headed the nanophotonics cluster at DTU Fotonik-Department of Photonics Engineering, formerly known as the Research Center COM, Technical University of Denmark. His current research interests are in the area of device physics, in particular nanophotonics devices, ultrafast devices for optical signal processing, slow light, noise in nonlinear devices, and quantum photonics. 